\title{
Dimorfismo sexual, ¿natural? Una reinterpretación crítica de las diferencias biológicas
}

\author{
Lucía Ciccia \\ Universidad Nacional Autónoma de México, Ciudad de México, México.
}

\begin{abstract}
Resumen
El presente trabajo expone una interpretación sexual dimórfica de las diferencias biológicas en la especie humana, que resulta de la lectura androcéntrica de los cuerpos que caracterizó la ciencia moderna. En contraste con esta lectura, se muestra cómo las prácticas sociales asociadas con los roles de género pueden traducirse en diferencias biológicas que "se ajustan" a una lectura dimórfica. Sobre la base de estos hechos, se propone que, si existen ciertos correlatos entre genitalidad y diferencias biológicas, los mismos no necesariamente son causados por los procesos de diferenciación sexual, sino por vínculos estadísticos dados por los estereotipos normativos de género. Las conductas implicadas en tales estereotipos se expresan biológicamente, y los roles de género crean muchas de las diferencias biológicas que hoy se asumen como naturalmente dimórficas.
\end{abstract}

Palabras clave: Sexo. Identidad de género. Caracteres sexuales.

\section{Resumo}

Dimorfismo sexual: natural? Uma reinterpretação crítica das diferenças biológicas

O presente trabalho expõe a interpretação sexual dimórfica das diferenças biológicas na espécie humana resultante da leitura androcêntrica dos corpos que caracterizou a ciência moderna. Em contraste com esta leitura, mostra-se como práticas sociais associadas aos papéis de gênero podem se traduzir em diferenças biológicas que se encaixam na leitura dimórfica. Com base nisso, propõe-se que, se existem certos correlatos entre genitalidade e diferenças biológicas, estes não são causados necessariamente por processos de diferenciação sexual, mas por vínculos estatísticos dados por estereótipos normativos de gênero. Os comportamentos envolvidos em tais estereótipos são biologicamente expressos, e os papéis de gênero criam muitas das diferenças biológicas hoje assumidas como naturalmente dimórficas.

Palavras-chave: Sexo. Identidade de gênero. Caracteres sexuais.

\section{Abstract \\ Sexual dimorphism: innate or acquired? A reinterpretation of biological differences}

This text argues that the dimorphic interpretation of biological differences in the human species results from an androcentric reading of bodies that have characterized modern science. In contrast to this perspective, the article shows how social practices associated with gender roles can produce biological differences that "adjust" themselves to a dimorphic reading. Based on these facts, we propose that if correlations between genitality and biological differences exists, they are not caused by the processes of sexual differentiation, but by statistical links given by normative gender stereotypes. The behaviors implied in such stereotypes are expressed biologically, and gender roles create many of the biological differences currently assumed as innate and sexually dimorphic.

Keywords: Sex. Gender identity. Sex characteristics.

La autora declara que no existe ningún conflicto de interés. 


\section{La lectura androcéntrica de las diferencias biológicas}

El método científico moderno supuso abandonar la teoría y el mundo de las formas abstractas platónicas para considerar la experimentación y el mundo concreto como el ideal del conocimiento ${ }^{1}$. Desde la epistemología feminista se identificó que este giro sustantivo en la manera de describir los fenómenos fue funcional a los intereses del sujeto androcéntrico (el varón cis -es decir, que no es trans-, heterosexual, blanco, propietario y occidental) en el marco de las sociedades preindustriales. Entre los intereses se destacan la secularización de la naturaleza, para intervenirla y dominarla por medio de la producción técnica y tecnológica, y la necesidad de polarizar los roles sociales, que circunscribe a las mujeres a las tareas de reproducción y cuidado ${ }^{1}$.

Tal escenario posibilitó un contexto propicio para albergar las ideas de Newton: su tesis mecanicista sirvió para reinterpretar la naturaleza viva y, exceptuándola de todo origen divino, habilitó su manipulación. El organismo humano también comenzó a ser objeto de exploración y experimentación, sirviendo como fuente de argumentos biológicos para justificar la reclusión de la mujer al ámbito privado. En efecto, se desarrolló un sistema de valores dicotómico, esencialmente jerárquico, cuya legitimidad se centró en una interpretación sexual dimórfica de las diferencias biológicas ${ }^{2}$. Los pares razón-emoción, objetividad-subjetividad, universal-particular, abstracto-concreto, activo-pasivo, público-privado, se correspondieron con el par masculino-femenino, respectivamente ${ }^{3}$.

Como describe Thomas Laqueur, un nuevo paradigma epistemológico desplazó el hasta entonces dominante modelo acerca de la anatomía de las semejanzas por otro que supuso una anatomía y una fisiología de lo inconmensurable ${ }^{4}$. Para finales del siglo XVIII, la idea de anatomías opuestas y complementarías caracterizó la interpretación de las diferencias entre varones y mujeres ${ }^{5}$.

En definitiva, la proyección del orden social dicotómico y jerárquico que comenzó a gestarse en la modernidad se justificó en una interpretación sexual dimórfica de las diferencias biológicas. Es decir, esta interpretación fue producto de los sesgos androcéntricos que impregnaron el discurso científico acerca de la diferencia sexual. De esta manera, las categorías macho-hembra se tornaron equivalentes a las categorías varón-mujer: dos organismos cualitativamente diferentes de acuerdo con los roles asociados con la reproducción.

Considerando que esos roles son el fundamento que da legitimidad a los estereotipos normativos de género y que pueden explicar las que hoy se consideran diferencias sexualmente dimórficas, este artículo se estructurará de la siguiente manera. El primer apartado muestra que en el ámbito biomédico persisten los mismos sesgos androcéntricos que caracterizaron al discurso científico acerca de la diferencia sexual durante la modernidad, pero actualizados sobre una lectura molecular posibilitada por el refinamiento técnico-tecnológico. Se describirán las consecuencias y los efectos negativos que pueden tener respecto de cómo se interpretan las prevalencias y el desarrollo de enfermedades en varones y mujeres.

El segundo apartado presenta críticas de epistemólogas feministas y científicas empiristas, además de exponer ciertas propuestas conceptuales para abonar a una reinterpretación de las diferencias biológicas que no recaiga en lecturas deterministas y esencialistas. Y el último concluye con la importancia de reconceptualizar nuestra biología y desandar los sesgos androcéntricos que aún prevalecen en el ámbito biomédico.

\section{La interpretación sexual dimórfica de las diferencias biológicas en los postulados actuales}

Durante el siglo XX, la interpretación dimórfica de las diferencias biológicas atravesó un proceso de molecularización debido a dos hechos fundamentales: el descubrimiento del gen SRY por el endocrinólogo francés Alfred Jost en 1940, y la consolidación de la neuroendocrinología como disciplina científica en $1959^{6,7}$. De tales acontecimientos se desprendieron los llamados "dogma clásico" y "teoría organizacional/activacional" (O/A).

El dogma clásico describe, en líneas generales, la diferenciación sexual en los mamíferos, incluso humanos, en términos cromosómicos:

El gen SRY, contenido en el cromosoma Y, se expresa en las células de los genitales primitivos, es decir, que aún no se encuentran sexualmente diferenciados, y compromete dicho tejido a un "destino 
testicular". Luego, los testículos secretan la hormona de inhibición mulleriana (cuya función es prevenir el desarrollo de los conductos que formarían el sistema reproductivo de la hembra) y la testosterona, que promueve el desarrollo de "estructuras masculinas" en otras partes del cuerpo ${ }^{7}$.

La teoría O/A extendió a los cerebros la interpretación dimórfica de la diferenciación sexual y planteó que a partir de un cerebro monomórfico (inicialmente "hembra") ocurre una diferenciación ("masculinización") causada por la testosterona. Dicha diferenciación, que organizaría permanentemente el cerebro de manera sexo-específica, se activaría en la vida posnatal y explicaría así las diferencias esenciales entre machos y hembras. Tanto el dogma clásico como la mencionada teoría sugieren que los procesos de diferenciación activos serían solo característicos del macho ${ }^{7}$.

Esta teoría, aplicada a la especie humana, asume la "masculinización" como causa explicativa de las diferencias cerebrales debidas al "sexo": la química hormonal y la mecánica fisiológica de la reproducción -ciclo de ovulación, eyaculación y erección-, y las llamadas conductas de género. Desde el discurso científico predominante suele asumirse que dichas conductas son aquellas no vinculadas directamente con la reproducción, sino ligadas a capacidades cognitivo-conductuales ${ }^{8}$. Es decir, el dimorfismo cerebral prenatal implicaría ciertas habilidades cognitivo-conductuales.

De acuerdo con el sistema de valores androcéntrico descrito en párrafos anteriores, el cerebro masculino estaría optimizado para aquellas habilidades y conductas "más valoradas"; en efecto, para las habilidades visual-espaciales, que implican la lectura de mapas y navegación (tareas vinculadas a la capacidad de abstracción), mientras que el cerebro femenino estaría optimizado para una habilidad denominada "fluidez verbal" (es decir, para hablar) 9 . Esta caracterización de los cerebros evidencia que la palabra "dimorfismo" se torna equivalente a -y legitimadora de- una distribución dicotómica, por tanto jerarquizada, de los roles sociales.

La interpretación acerca de un camino monomórfico en que "luego" ocurre la masculinización y des-feminización del macho-varón se encuentra en estrecha vinculación con el par dicotómico activo-pasivo (macho-hembra, respectivamente). Esta vinculación fue respaldada de manera explícita por las diversas disciplinas científicas que emergieron durante el siglo XIX, como la embriología y la craneología. Tales disciplinas afirmaron sin evidencia empírica que llegar a ser macho requería de una complejización/especialización ${ }^{10}$. En definitiva, los postulados planteados por el dogma clásico y por la teoría O/A reflejan el anacronismo de los sesgos androcéntricos que implicaron interpretar las diferencias biológicas en el marco de una lectura dicotómica y jerárquica de los cuerpos.

\section{Las consecuencias de una interpretación}

dimórfica de las diferencias biológicas en el ámbito biomédico

La lectura jerárquica que subyace a la interpretación sexual dimórfica de las diferencias biológicas es proyectada en la omisión de hembras y mujeres en los protocolos de investigación básicos, preclínicos y clínicos ${ }^{11}$. Por eso, el Instituto Nacional de Salud Norteamericano ( $\mathrm{NIH}$, por sus siglas en inglés), la Comisión Europea y el Instituto Canadiense de Investigación en Salud comenzaron a exigir que las investigaciones financiadas por ellos incluyan tanto machos como hembras (en estudios sobre animales, tejidos y/o células) en sus diseños experimentales, y que el sexo se considere una variable biológica en sus análisis, salvo pocas excepciones ${ }^{12}$.

Ciertos trabajos comprueban los sesgos que resultan de omitir a la hembra e interpretar los procesos de diferenciación genital y cerebral tomando al macho como único eje de referencia y material acabado que "contiene" a la hembra. En este sentido, si bien se ha constatado que el gen SRY inicia la diferenciación testicular en machos, también en las hembras existen genes encargados de iniciar la diferenciación ovárica. Es decir, existe una diferenciación genital activa en la hembra. Este hecho se observó no solo en mamíferos, sino también en pájaros e incluso en tortugas, en las cuales la determinación del sexo depende de la temperatura ${ }^{13}$.

En relación con los cerebros, aunque se corroboró que existe un proceso de masculinización y desfeminización en ratones machos ${ }^{14}$, esto no es equivalente a legitimar la idea de un cerebro monomórfico a partir del cual ocurre la masculinización. En contraste, un estudio realizado en ratones hembras encontró un proceso de feminización y desmasculinización ${ }^{15}$. En otras palabras, los resultados obtenidos sugieren la existencia de 
un cerebro "dual", con la presencia simultánea de circuitos de machos y hembras en cada organismo, implicando también la presencia de patrones de expresión sexual activos, tanto respecto de la montada del macho como de la lordosis (curvatura de la columna) de la hembra ${ }^{15}$.

Al sugerir que existen procesos paralelos de diferenciación genital y cerebral entre machos y hembras, y no una "complejización" hasta llegar a ser macho, estos dos trabajos ${ }^{14,15}$ visibilizan los sesgos androcéntricos decimonónicos presentes en la interpretación de los procesos de diferenciación genital y cerebral. Así se abre una grieta para comenzar a reinterpretar tales procesos en el ámbito biomédico.

Cabe resaltar que la interpretación dimórfica de las diferencias biológicas es en sí misma fruto del orden social dicotómico en la modernidad. Es decir, los sesgos androcéntricos no se diluyen solo validando que existen procesos paralelos en los mecanismos de diferenciación. En cambio, desestabilizar de manera estructural dichos sesgos implica cuestionar la supuesta naturaleza rígida y dimórfica de los procesos de diferenciación.

La inclusión de hembras y mujeres en los protocolos de investigación es una condición necesaria para desandar los sesgos androcéntricos que caracterizan la producción de conocimiento biomédico. Sin embargo, no es suficiente. Además, debe considerarse la manera en que se interpreta tanto dicha inclusión como los resultados obtenidos a partir de un estudio que incorpore "ambos sexos". En relación con la forma de inclusión, debe analizarse cómo la categoría macho es caracterizada en tanto modelo experimental.

En este sentido, es curioso que la justificación para seleccionar solo machos en los estudios experimentales sea la de evitar las fluctuaciones hormonales de las hembras. Es decir, reportar el estatus hormonal de las hembras se convierte en un obstáculo que solo complejiza el análisis de datos (y, por tanto, la obtención de resultados publicables a corto plazo) si no es específicamente el objetivo del estudio en cuestión ${ }^{10}$. Paradójicamente, gran parte de los estudios que no son reproducibles se debe a un mal reporte del estatus hormonal de los machos: la testosterona también fluctúa, presentando, por ejemplo, ritmos estacionales y circadianos ${ }^{16}$.
Sin embargo, al extrapolarse la fisiología animal no humana a la especie humana, la historia de la endocrinología ha instalado la idea de un vínculo causal entre la fluctuación hormonal de las hembras-mujeres y "su" inestabilidad emocional ${ }^{17}$. $\mathrm{Ni}$ siquiera las preguntas de investigación orientadas a vincular la noción de "fluctuación" con la hormona testosterona evidencian el fuerte arraigo de una lectura androcéntrica -por tanto biologicista- de las diferencias: la supuesta "estabilidad emocional del varón" se justifica en la "estabilidad hormonal" del macho. La estabilidad, a su vez, se asocia con la predisposición innata para ejercer la "objetividad" y la "neutralidad". Este es otro ejemplo de cómo la descripción dimórfica de las diferencias biológicas se ajusta a una organización social dicotómica y jerárquica de los cuerpos.

Respecto de los resultados, es necesario cuestionar qué se interpreta que las categorías macho y hembra nos "informan" en un estudio determinado. Como la interpretación dimórfica supone dos categorías cualitativamente diferentes entre sí, a la vez homogéneas "hacia dentro" de cada una de ellas, se considera que incorporar machos y hembras equivale a introducir una variable biológica. Es decir, en primer lugar, se comparan; en segundo lugar, se asume que las posibles diferencias encontradas reflejan diferencias biológicas innatas, fijas e inmutables. Este hecho se extrapola también a la especie humana, como lo demuestra Janine Clayton:

Para apreciar la consideración del sexo como una variable biológica, es necesario definir y distinguir el sexo del género. El "sexo" se origina del complemento cromosómico sexual de un organismo: cromosomas $X X$ o $X Y$ en humanos, y se refleja en los órganos reproductivos. Cada célula tiene un sexo. El sexo de uno afecta todos los aspectos del funcionamiento fisiológico, no solo las secreciones hormonales. Aunque el sexo de uno también puede afectar el comportamiento, otros factores, sociales y culturales, también pueden influir en el comportamiento. Por lo tanto, el término "género" se refiere a rasgos sociales, culturales y psicológicos vinculados a hombres y mujeres humanos a través del contexto social ${ }^{18}$.

Para Clayton ${ }^{19}$, directora de la Oficina de Investigación sobre la Salud de la Mujer (perteneciente al NIH), la caracterización sexual dimórfica 
es un hecho, por lo cual se entiende el sexo como una variable biológica fundamental. Desde esta perspectiva, cualquier diseño experimental con fines biomédicos debería partir del criterio de agrupación varón-mujer, reflejo de las categorías macho-hembra, respectivamente, para la búsqueda de diferencias biológicas esenciales.

Como afirma la autora ${ }^{19}$, suele asumirse que existen diferencias sexualmente dimórficas en las secreciones hormonales de las justamente etiquetadas "hormonas sexuales": el estradiol, la progesterona y la testosterona. Aunque todas las personas portamos todas las "hormonas sexuales", se condujeron a estudiar solo estradiol y progesterona en mujeres, y testosterona en varones ${ }^{20}$.

Apenas en estos últimos años emergen trabajos sobre las "hormonas sexuales" en mujeres y en varones. Los resultados sugieren que los niveles promedio de estradiol y progesterona no difieren entre varones y mujeres, lo cual diluye la idea de dimorfismo sexual para dichas hormonas. Aunque se sigue encontrando, en promedio, un mayor nivel de testosterona en varones en comparación con mujeres, esta diferencia es mucho menor de la que se supone, y existen, además, grandes solapamientos ${ }^{21}$. Es decir, las concentraciones de testosterona son variables, pueden o no existir diferencias, dependiendo de qué personas compongan la muestra del estudio en cuestión.

En resumen, una interpretación sexual dimórfica de las diferencias biológicas conduce a sesgos tanto en la manera en que los modelos experimentales son caracterizados (el macho en tanto "biología ideal") como en los diseños experimentales (omisión de las hembras o su inclusión, conceptualizándola una variable biológica). Esto repercute directamente en cómo se interpreta la manera de enfermar: se la asocia fundamentalmente al sexo, mientras que el género, es decir, las prácticas sociales de acuerdo con una genitalidad, se considera periférico.

\section{Categorías conceptuales para flexibilizar nuestra biología}

La necesidad de distinción entre los conceptos de "sexo" y "género", que en la literatura biomédica suelen utilizarse de manera intercambiable, llevó al NIH a impartir un curso online sobre el tema. El empleo de "sexo" y "género" como sinónimo no se debe solo a que en el inglés vernáculo los términos son literalmente sinónimos. En cambio, es también porque para el discurso científico predominante el género resulta del sexo. Es decir, se entiende que existe un vínculo causal entre biología (sexo) y conducta (género). De esta manera, sexo y género son traducidos como sinónimos en el lenguaje biológico.

Sin embargo, la idea de género promovida por el NIH sugiere que nuestro cuerpo es un sistema acabado al cual terminan por afectarlo -en términos aditivos- nuestras prácticas sociales generizadas. Como señalan Shattuck-Heidorn y Richardson, el ejemplo que utilizan para mostrar cómo el género puede impactar en nuestra biología es el escenario simple y estereotipado del uso de tacones altos sobre las articulaciones de la rodilla ${ }^{22}$.

Desde esta perspectiva, los estereotipos de género parecieran no encarnarse, sino representar disfraces superficiales, "medibles" y "observables" de manera lineal. En contraste, el sexo representaría las diferencias "profundas" entre varones y mujeres, interpretándose como una variable biológica dimórfica precisa y constante. Se aplica aquí la idea de profundidad para evidenciar que esta lectura se sostiene al asumir que detrás del varón hay un macho, y detrás de la mujer una hembra.

En contraposición a esta conceptualización rígida y dimórfica para caracterizar las diferencias biológicas, las investigadoras que conforman la NeuroGenderings Network, una red interdisciplinar de prestigiosas investigadoras críticas respecto del discurso neurocientífico predominante, complejizan la forma de interpretar el sexo. Muchas de ellas explicitan que, si bien es recomendable que hembras y mujeres se incorporen en cualquier estudio que hoy se realiza solo en machos y varones, tal incorporación no supone necesariamente introducir el sexo como una variable biológica. En cambio, se trata de tener una mayor representatividad de la especie de la que se tendría si solo se estudiara machos o hembras ${ }^{23}$.

Además, plantean la necesidad de considerar otros factores que varían con el sexo. En este sentido, en contraste con la idea de género sugerida por el $\mathrm{NIH}$, enfatizan que la alta plasticidad que caracteriza nuestra especie convierte al género en algo más que un factor superficial. Aparece entonces la idea de una biología flexible que dialoga y se retroalimenta con/de nuestras prácticas de género. Para 
visibilizar este diálogo hay dos autoras, entre otras, que introdujeron conceptos fundamentales ${ }^{24,25}$.

La primera es Nancy Krieger ${ }^{24}$, quien desarrolla la idea de expresión biológica en el marco de la epidemiología social y alude a cómo las prácticas sociales de género, que implican desigualdad económica, pueden afectar nuestra salud. En otras palabras, al caracterizar la desigualdad socioeconómica como factor fundamental para la expresión diferenciada de una enfermedad, Krieger habla de la expresión biológica generizada. En la próxima sección se profundizará el alcance de este concepto.

La segunda autora es Anelis Kaiser ${ }^{25}$, integrante fundadora de la NeuroGenderings Network, quien propuso incorporar la noción de sexo/género en el campo de las neurociencias para evidenciar que no es posible "desagregar" en el cerebro los factores puramente biológicos de los factores asociados a nuestra experiencia social generizada. La autora recomienda que los estudios cerebrales orientados a la búsqueda de diferencias entre varones y mujeres no se refieran a "diferencias de sexo", sino a "diferencias de sexo/género" ${ }^{25}$. Por supuesto, esta idea es extensible a todo nuestro organismo.

\section{Expresión biológica generizada: el cerebro como punto de partida}

La investigadora israelí Daphna Joel y colaboradores ${ }^{26}$ mostraron la invalidez de caracterizar los cerebros según las categorías varón-mujer, debido a que la alta variabilidad existente entre los cerebros de mujeres, por un lado, y entre los cerebros de varones, por otro, sería igual a la alta variabilidad existente entre cerebros de mujeres y varones. Joel y colaboradores ${ }^{26}$ proponen entonces la hipótesis del cerebro mosaico, que equivaldría a conceptualizar cada cerebro como una combinación única de factores. Este tipo de hipótesis abre el interrogante acerca de si la agrupación de acuerdo con las categorías varón-mujer para buscar "diferencias" no resultaría en falsos positivos.

Aunque debe haber participantes tanto mujeres como varones en cada estudio de la estructura y función del cerebro humano para representar mejor la variabilidad total de nuestra especie, el uso de la categoría de sexo como una variable para analizar los resultados de tales estudios no debe ser el predeterminado (...) [esto] conduciría a la detección de diferencias aleatorias entre los grupos de varones y mujeres en el estudio ${ }^{27}$.

Asimismo, en caso de encontrarse -y que fueran válidas- diferencias entre varones y mujeres para un parámetro cerebral dado (en términos de estructura y/o función), las mismas no deberían interpretarse con el peso de la causalidad. En cambio, tendría que evaluarse la contribución de nuestras prácticas sociales sobre tales diferencias. Debido a su alta plasticidad, el cerebro es el órgano paradigmático para comprender cómo las prácticas sociales pueden modificar nuestro organismo:

Ahora está claro que la organización funcional e incluso estructural del sistema nervioso humano es un proceso continuo y dinámico que persiste a lo largo de la vida. La plasticidad dependiente de la experiencia se ha demostrado una y otra vez en la adquisición de habilidades tan diversas como la interpretación musical, el baloncesto, el baile, la conducción de taxis y el malabarismo ${ }^{28}$.

En definitiva, los trabajos de las investigadoras de la red muestran que la caracterización dimórfica de los cerebros es inválida, dejando planteada la necesidad de desarrollar nuevos criterios de agrupación.

De lo anterior, se puede caracterizar que las prácticas de género se entrenan, se vuelven ejercicios que corporizamos por medio de hábitos que aprendemos, memorizamos, producimos y reproducimos a diario. Por ello, se propone definir la conexión que existe entre genitalidad y género como estadística. Una conexión estadística se explica más bien por los estereotipos normativos de género que por una determinación biológica.

En este sentido, se aplica la idea de expresión biológica generizada, que puede utilizarse para describir un vínculo estadístico entre genitalidad y ciertas diferencias biológicas que hoy se observan entre varones y mujeres. Se trata de un vínculo estadístico entre nuestra genitalidad y nuestra expresión biológica generizada, ya no solo en un sentido sociológico como el propuesto por Krieger ${ }^{24}$, sino también ontológico. Es decir, la correlación normativa entre nuestra genitalidad y el género que nos asignan al nacer implica la corporización de nuestras prácticas generizadas, las cuales terminan por expresarse biológicamente. 


\section{Más allá de los cerebros}

La idea de vínculo estadístico para interpretar las correlaciones entre genitalidad y expresión biológica se puede extender a otros órganos y procesos fisiológicos. Por ejemplo, en el ámbito farmacológico, reducir las exigencias a la mera inclusión del sexo como variable biológica en los diseños experimentales ha sido fuente de críticas porque invisibiliza, o trata como periféricos, factores capaces de afectar la metabolización o aclareamiento de fármacos. Tales factores se relacionan con hábitos sumamente generizados: la actividad física, la alimentación y el consumo de componentes bioactivos, como tabaco, café o alcohol, entre otros ${ }^{29}$. El peso corporal también afecta la velocidad de eliminación de ciertos fármacos, tal como se comprobó para el hipnótico zolpidem ${ }^{30}$. Debido a que todos estos factores tienen efectos centrales en la farmacocinética, se convierten en variables fundamentales.

En este sentido, es necesario generar herramientas para indagar cuáles factores genéticos y sociales -o cómo los sociales pueden afectar los genéticos- contribuyen a metabolizar un determinado fármaco. Así, si un estudio buscara diferencias entre mujeres y varones en la velocidad de metabolización de un fármaco, y se observara, por ejemplo, que en las mujeres la velocidad es menor en comparación con los varones, este hecho no significa que existan diferencias ligadas -en última instancia- al sexo. En cambio, podrían explicarse por ciertos hábitos generizados que afectan la velocidad de metabolización. En este caso, la correlación entre genitalidad y metabolización de fármaco debería entenderse como un vínculo estadístico, y no causal, debiéndose contextualizar otras características y hábitos culturales de los participantes del estudio.

Por otro lado, la idea sugerida por Clayton ${ }^{19}$, de que las concentraciones hormonales son dimórficas, no solo es cuestionada por los solapamientos antes descritos, sino que hay hallazgos en el ámbito de la neuroendocrinología social que desafían la creencia (dominante en el ámbito biomédico) de que el sexo define las concentraciones hormonales. En lugar de partir de las concentraciones hormonales para luego asociarlas con ciertas conductas -metodología clásica de la neuroendocrinología comportamental-, esta disciplina estudia los efectos que el ambiente/contexto social tiene sobre la regulación hormonal. Así se observó que el rechazo social incrementa los niveles de progesterona $^{31}$, y que contextos de dominancia incrementan tanto el estradiol como la progesterona ${ }^{32}$. Es decir, las concentraciones hormonales varían como consecuencia de nuestras prácticas sociales.

En la misma línea, la investigadora Van Anders ${ }^{20}$ demostró que factores no genéticos influyen fuertemente en las concentraciones de testosterona. Además de los ritmos estacionales y circadianos, también ciertos roles sociales, fuertemente generizados, pueden afectar las concentraciones. Por ejemplo, independientemente de si se trata de varones o mujeres, ante contextos de competencia hay un incremento en los niveles de testosterona, mientras que ante actividades asociadas al cuidado se observa una reducción de los mismos ${ }^{20}$. A la luz de estos hallazgos, se abre el siguiente interrogante: ¿a qué se debe la pequeña diferencia que, en promedio, se observa entre varones y mujeres en la concentración de testosterona?

Los estudios descritos en esta sección muestran que la idea de sexo como dimórfico resulta inválida en términos cerebrales y en relación, "al menos", con los procesos farmacocinéticos y las "hormonas sexuales". Habría que analizar qué otros parámetros biológicos, que continúan considerados sólidos legitimadores de una interpretación sexual dimórfica de las diferencias biológicas, no resultan en realidad de una regulación biosocial normada por los estereotipos de género.

Tal escenario sugiere que en el ámbito biomédico las categorías varón-mujer deberían remitir a expresiones biológicas que materializan un vínculo estadístico normativo. En otras palabras, los estereotipos de género pueden explicar muchas de las diferencias biológicas que actualmente se observan entre varones y mujeres.

Si bien excede los límites de este trabajo, cabe plantear que las prácticas sociales no solo se encuentran generizadas, sino también atravesadas por otras categorías normativas, como las asociadas con los procesos de racialización. Tales categorías intersectan y conviven en un mismo cuerpo. En consecuencia, comprender la manera en que la experiencia social repercute en nuestro organismo requiere de una perspectiva interseccional.

En esta línea, un caso ilustrativo es el trabajo reciente de Krieger, Jahn y Waterman ${ }^{33}$, quienes encontraron una asociación entre la incidencia de un 
tipo de cáncer de mama y las leyes de Jim Crow -la segregación racial legal practicada en 21 estados de Estados Unidos hasta 1964. Las autoras mostraron que hubo mayor incidencia de este cáncer en mujeres negras nacidas antes de 1964, en comparación con las que nacieron después, mientras que esta diferencia no se observó en las mujeres blancas ${ }^{33}$.

\section{Consideraciones finales}

Desde la modernidad, el orden social jerárquico y dicotómico se justificó biológicamente sobre una interpretación sexual dimórfica. Aunque el discurso científico predominante actual sostenga dicha interpretación, la biología molecular, lejos de reafirmarla, muestra su anacronismo. Así, la evaluación de las diferencias entre varones y mujeres, dando por hecho un dimorfismo sexual, puede conducir a resultados sesgados, que obstaculizan una verdadera comprensión de los mecanismos que explican las prevalencias y el desarrollo de enfermedades. La singular plasticidad que nos caracteriza como especie, condicionada estructuralmente por los roles asociados al género, implica un gran impacto de nuestras prácticas sociales en nuestra expresión biológica.

Desde una perspectiva sexo-genérica, para evaluar dicho impacto es necesario desplazar la idea de vínculo causal entre sexo y género por la noción de vínculo estadístico. Si existen diferencias biológicas para cierto parámetro entre varones y mujeres, esta noción habilita conceptualizarlas en el marco de las prácticas sociales embebidas en los estereotipos de género.

Como mostró el trabajo de Van Anders ${ }^{20}$, los hábitos generizados pueden incrementar o disminuir los niveles de testosterona. En este sentido, es fundamental evaluar los alcances de nuestras prácticas generizadas sobre nuestra expresión biológica. Por ejemplo, ¿cómo afectan nuestras prácticas generizadas la expresión génica relativa a los cromosomas que, como las hormonas, también son etiquetados de "sexuales"?

Debido a que la interpretación sexual dimórfica de las diferencias biológicas resulta de una lectura androcéntrica moderna de los cuerpos, es necesario revisar y analizar los presupuestos e hipótesis que guían los estudios biomédicos orientados a la búsqueda de diferencias sexuales. Cabe subrayar que dicha lectura alimenta la idea de una biología rígida, determinada y binaria, que no se ajusta a nuestras realidades biológicas: desde la expresión cromosómica, pasando por la expresión genital, hasta la expresión cerebral, nuestra diversidad y dinamismo biológico trasciende la dicotomía reduccionista.

Para no sobrevalorar la contribución de factores genéticos en nuestra expresión biológica, se debe comenzar a generar métodos que visibilicen y complejicen las variables sociales que, a su vez, pueden afectar factores genéticos. Asimismo, se debe situar nuestra expresión biológica en el marco de los actuales estereotipos de género. Pero tales estereotipos no deben universalizarse, sino complejizarse desde una perspectiva geopolítica. Es decir, no es lo mismo el estereotipo de género femenino en el mundo anglosajón, de donde proviene la mayoría de los estudios aquí citados, que en América Latina. Es necesario producir conocimiento en esta dirección teniendo presentes las condiciones estructurales locales.

En definitiva, cuando se observan diferencias biológicas entre varones y mujeres no se analiza un vínculo causal, ahistórico y atemporal, entre genitalidad y dichas diferencias, sino que se permite desarrollar nuevas estrategias epistémicas y metodológicas para comprender cómo funciona nuestro organismo y los procesos de diferenciación a él asociados, así como habilita generar otras herramientas para perevenir y tratar enfermedades.

\section{Referencias}

1. Fox Keller E. Reflexiones sobre género y ciencia. Valencia: Alfons el Magnánim; 1991.

2. Ciccia L. La ficción de los sexos: hacia un pensamiento neuroqueer desde la epistemología feminista [tese] [Internet]. Buenos Aires: Universidad de Buenos Aires; 2017 [acesso 21 maio 2020]. Disponível: https://bit.ly/2OsBXN9 
3. Maffía D. Contra las dicotomías: feminismo y epistemología crítica [Internet]. Buenos Aires: Universidad de Buenos Aires; [s.d.] [acesso 21 maio 2020]. Disponível: https://bit.ly/3d110ks

4. Laqueur T. La construcción del sexo: cuerpo y género desde los griegos hasta Freud. Madrid: Cátedra; 1994. p. 24.

5. Schiebinger L. ¿Tiene sexo la mente? Las mujeres en los orígenes de la ciencia moderna. Madrid: Cátedra; 2004.

6. Wallen K. Organizational hypothesis: reflections on the 50th anniversary of the publication of Phoenix, Goy, Gerall, and Young (1959). Horm Behav [Internet]. 2009 [acesso 20 jun 2020];55(5):561-5. DOI: 10.1016/ j.yhbeh.2009.03.009

7. Arnold AP, Xu J, Grisham W, Chen X, Kim YH, Itoh Y. Minireview: sex chromosomes and brain sexual differentiation. Endocrinol [Internet]. 2004 [acesso 21 maio 2020];145(3):1057-62. p. 1057. Tradução livre. DOI: 10.1210/en.2003-1491

8. Shattuck-Heidorn H, Richardson SS. Sex/gender and the biosocial turn. Neurogenderings [Internet]. 2019 [acesso 21 maio 2020];15(2). Disponível: https://bit.ly/2Z3MTmx

9. Ciccia L. La dicotomía de los sexos puesta en jaque desde una perspectiva cerebral. Descentrada [Internet]. 2018 [acesso 21 maio 2020];2(2):e052. Disponível: https://bit.ly/3ddeCJR

10. Ciccia L. Premio Anual de Bioética 2017: $1^{\text {a }}$ mención: el sexo y el género como variables en la investigación biomédica y la práctica clínica [Internet]. Buenos Aires: Fundación Dr. Jaime Roca; 2017 [acesso 10 fev 2021]. Disponível: https://bit.ly/2Z5YjpG

11. Klein SL, Schiebinger L, Stefanick ML, Cahill L, Danska J, Vries GJ et al. Opinion: sex inclusion in basic research drives discovery. Proc Natl Acad Sci USA [Internet]. 2015 [acesso 21 maio 2020];112(17):5257-8. DOI: $10.1073 /$ pnas.1502843112

12. Kleinherenbrink AV. The politics of plasticity: sex and gender in the 21st century brain [tese] [Internet]. Amsterdam: University of Amsterdam; 2016 [acesso 21 maio 2020]. Disponível: https://bit.ly/3jCLv3A

13. Piprek RP. Molecular mechanisms underlying female sex determination: antagonism between female and male pathway. Folia Biol (Kraków) [Internet]. 2009 [acesso 21 maio 2020];57(3-4):105-13. DOI: 10.3409/ fb57_3-4.105-113

14. McCarthy MM, Nugent BM. At the frontier of epigenetics of brain sex differences. Front Behav Neurosci [Internet]. 2015 [acesso 22 abr 2019];9:221. DOI: 10.3389/fnbeh.2015.00221

15. McCarthy MM, Pickett LA, VanRyzin JW, Kight KE. Surprising origins of sex differences in the brain. Horm Behav [Internet]. 2015 [acesso 21 maio 2020];76:3-10. DOI: 10.1016/j.yhbeh.2015.04.013

16. Becker J, Arnold AP, Berkley KJ, Blaustein JD, Eckel LA, Hampson E et al. Strategies and methods for research on sex difference in brain and behavior. Endocrinol [Internet]. 2005 [acesso 21 maio 2020];146(4):1650-73. DOI: 10.1210/en.2004-1142

17. Hayward JA. Historia de la medicina. Buenos Aires: Biblioteca Actual; 1989.

18. Clayton J. Applying the new SABV (sex as a biological variable) policy to research and clinical care. Physiol Behav [Internet]. 2018 [acesso 21 maio 2020];187:2-5. p. 2. Tradução livre. DOI: 10.1016/ j.physbeh.2017.08.012

19. Clayton J. Op. cit.

20. Van Anders SM. Beyond masculinity: testosterone, gender/sex, and human social behavior in a comparative context. Front Neuroendocrinol [Internet]. 2013 [acesso 21 maio 2020];34(3):198-210. DOI: 10.1016/ j.yfrne.2013.07.001

21. Liening SH, Stanton SJ, Saini EK, Schultheiss OC. Salivary testosterone, cortisol, and progesterone: two-week stability, interhormone correlations, and effects of time of day, menstrual cycle and oral contraceptive use on steroid hormone levels. Physiol Behav [Internet]. 2010 [acesso 21 maio 2020];99(1):8-16. DOI: 10.1016/j.physbeh.2009.10.001

22. Shattuck-Heidorn H, Richardson SS. Op. cit. Tradução livre. 
23. Rippon G, Jordan-Young R, Kaiser A, Joel D, Fine C. Journal of Neuroscience Research policy on addressing sex as a biological variable: comments, clarifications, and elaborations. J Neurosci Res [Internet]. 2017 [acesso 21 maio 2020];95(7):1357-9. DOI: 10.1002/jnr.24045

24. Krieger N. A glossary for social epidemiology. J Epidemiol Community Health [Internet]. 2001 [acesso 21 maio 2020];55(10):693-700. DOI: 10.1136/jech.55.10.693

25. Keiser A. Re-conceptualizing "sex" and "gender" in the human brain. Z Psychol [Internet]. 2012 [acesso 21 maio 2020];220(2):130-6. DOI: 10.1027/2151-2604/a000104

26. Joel D, Berman Z, Tavorc I, Wexlerd N, Gaber O, Stein Y et al. Sex beyond the genitalia: the human brain mosaic. Proc Natl Acad Sci USA [Internet]. 2015 [acesso 21 maio 2020];112(50):15468-73. DOI: 10.1073/ pnas.1509654112

27. Joel D, Persico A, Salhov M, Berman Z, Oligschläger S, Meilijson I, Averbuch A. Analysis of human brain structure reveals that the brain "types" typical of males are also typical of females, and vice-versa. Front Hum Neurosci [Internet]. 2018 [acesso 21 maio 2020];12:399. p. 16. Tradução livre. DOI: 10.3389/ fnhum.2018.00399

28. Fine C, Jordan-Young R, Kaiser A, Rippon G. Plasticity, plasticity, plasticity... and the rigid problem of sex. Trends Cogn Sci [Internet]. 2013 [acesso 21 maio 2020];17(11):550-1. p. 550. Tradução livre. DOI: 10.1016/ j.tics.2013.08.010

29. Schiebinger L, Stefanick ML. Gender matters in biological research and medical practice. J Am Coll Cardiol [Internet]. 2016 [acesso 21 maio 2020];67(2):136-8. DOI: 10.1016/j.jacc.2015.11.029

30. Richardson SS, Reiches M, Shattuck-Heidorn H, LaBonte ML, Consoli T. Opinion: focus on preclinical sex differences will not address women's and men's health disparities. Proc Natl Acad Sci USA [Internet]. 2015 [acesso 21 maio 2020];112(44):13419-20. DOI: 10.1073/pnas.1516958112

31. Duffy KA, Harris LT, Chartrand TL, Stanton SJ. Women recovering from social rejection: the effect of the person and the situation on a hormonal mechanism of affiliation. Psychoneuroendocrinology [Internet]. 2017 [acesso 21 maio 2020];76:174-82. DOI: 10.1016/j.psyneuen.2016.11.017

32. Stanton SJ, Schultheiss OC. Basal and dynamic relationships between implicit power motivation and estradiol in women. Horm Behav [Internet]. 2007 [acesso 21 maio 2020];52(5):571-80. DOI: 10.1016/ j.yhbeh.2007.07.002

33. Krieger N, Jahn JL, Waterman PD. Jim Crow and estrogen-receptor-negative breast cancer: US-born black and white non-Hispanic women, 1992-2012. Cancer Causes Control [Internet]. 2017 [acesso 21 maio 2020];28:49-59. DOI: 10.1007/s10552-016-0834-2

Lucía Ciccia - Doctora - lucia_ciccia@cieg.unam.mx

(D) 0000-0002-0644-883X

Correspondencia

Torre II de Humanidades, piso 14, oficina 6, Circuito Interior, Ciudad Universitaria, Coyoacán CP 04510. Ciudad de México, México.

Recibido: 8.10.2019

Revisado: $\quad 5.1 .2021$

Aprobado: 12.1.2021 